\title{
A case of congenital tuberculosis-infected twins born to a mother with asymptomatic tuberculosis during pregnancy
}

\author{
Jiwon Park, Kyoung Sim Kim, Yong Wook Kim, Eun Young Kim, Young Kim, Hae In Jang, \\ Hyoung Min Cho
}

Department of Pediatrics, Kwangju Christian Hospital, Gwangju, Korea

Received August 31, 2021

Revised October 18, 2021

Accepted October 27, 2021

Corresponding author

Hyoung Min Cho

Department of Pediatrics, Kwangju

Christian Hospital, 37 Yangnim-ro,

Nam-gu, Gwangju 61661, Korea

Tel: +82-62-650-5045

Fax: +82-62-650-5040

E-mail: drcho92@hanmail.net

ORCID:

https://orcid.org/0000-0003-1263-5292
Congenital tuberculosis infection is very rare, but fatal. Congenital tuberculosis should always be considered among the many causes of pneumonia, especially if the newborn has pneumonia that does not respond to treatment. We report a case of a premature infant born to a mother who had no evidence of tuberculosis infection. The patient was the first of the twins born by cesarean section at 30 weeks and 1 day of gestation. The $21^{\text {st }}$ day after birth, the patient developed apnea, tachycardia, and tachypnea. The medical staff assumed sepsis and bacterial pneumonia. Despite antibiotic treatment, the patient's condition worsened. On the $63^{\text {rd }}$ day after birth, the mother of the infant visited the emergency room complaining of fever and decreased consciousness and was diagnosed with tuberculous meningitis. Mycobacterium tuberculosis was detected in the AFB stain, TB PCR, and TB culture.

Keywords: Congenital tuberculosis; Newborn; Premature; Pulmonary arterial hypertension; $\mathrm{Ap}-$ nea

\section{INTRODUCTION}

Congenital tuberculosis infection is very rare but fatal [1]. Among 215 countries and territories, the Republic of Korea ranked 87 th in the rate of tuberculosis incidence and 102nd in the rate of tuberculosis mortality. It has the highest incidence rate (59 per 100,000 population) and the second highest tuberculosis mortality rate (4.0 per 100,000 population) among OECD countries [2]. Although the prevalence of tuberculosis is decreasing in developed countries, the prevalence of tuberculosis remains high among economically low-income people and immigrants [3]. Given the high incidence rate, pediatricians need to consider the possibility of congenital tuberculosis. Congenital tuberculosis should always be considered among the many causes of pneumonia, especially if the newborn has pneumonia that does not respond to treatment.

We report a case of a premature infant born to a mother who had no evidence of tuberculosis infection. The infant developed pneumonia from the 3 rd week of age, did not respond well to treatment, and was diagnosed with congenital tuberculosis at 7 weeks of age.

\section{CASE REPORT}

The patient was the first of the twins born by cesarean

This is an Open Access article distributed under the terms of the Creative Commons Attribution Non-Commercial License (http://creativecommons.org/licenses/by-nc/4.0) which permits unrestricted noncommercial use, distribution, and reproduction in any medium, provided the original work is properly cited.

Copyright (c) Medical Biological Science and Engineering. 
section at 30 weeks and 1 day of gestation in another tertiary hospital. The mother was pregnant by in vitro fertilization and embryo transfer (IVT-ET). The twins had 2 amnions, 2 chorions, 2 placentas. The placenta biopsy was no special findings other than focal placental infarct. The patient's parents were Ugandan. Uganda is one of the TB/ HIV high-burden countries defined by WHO for the period 2016-2020 [4]. BCG vaccination has been implemented in Uganda since 1960, but the mother's BCG vaccination history is not accurately remembered. And they were illegal immigrants. The mother showed no signs of suspicion of tuberculosis and normal chest radiographs were obtained during the cesarean section. The patient was female with a birth weight of $1,590 \mathrm{~g}$. The Apgar scores were 9 points at 1 minute and 10 points at 5 minutes. The twins were transferred to our hospital on the 15th day after birth because of hospital expenses. Our hospital was a secondary hospital, and the cost was lower than that of the hospital that where the patient was previously hospitalized.

On the first day of hospitalization, the patient had normal chest radiographic findings (Fig. 1A) and had no respiratory symptoms such as dyspnea, apnea, or tachypnea. The respiratory rate was 60 times/min. The initial laboratory tests showed a hemoglobin concentration of $15.3 \mathrm{~g} / \mathrm{dL}$, white blood cell count of $10,600 / \mathrm{mm}^{3}$, platelet count of 289,000 / $\mathrm{mm}^{3}$, and C-reactive protein (CRP) of $0.0 \mathrm{mg} / \mathrm{dL}$. On the $6 \mathrm{th}$ day of hospitalization, the 21st day after birth, the patient developed apnea, tachycardia, and tachypnea. The heart rate was $163 / \mathrm{min}$, and the respiratory rate was $85 / \mathrm{min}$. No decrease in blood pressure was observed. The patient looked very sick and showed moaning. On physical examination, the patient breathed rapidly, and crackles were auscultated in both lung fields. Apnea and desaturation worsened, nasal continuous positive airway pressure treatment was started. Laboratory tests performed on the same day showed a hemoglobin concentration of $12.6 \mathrm{~g} / \mathrm{dL}$, white blood cell count
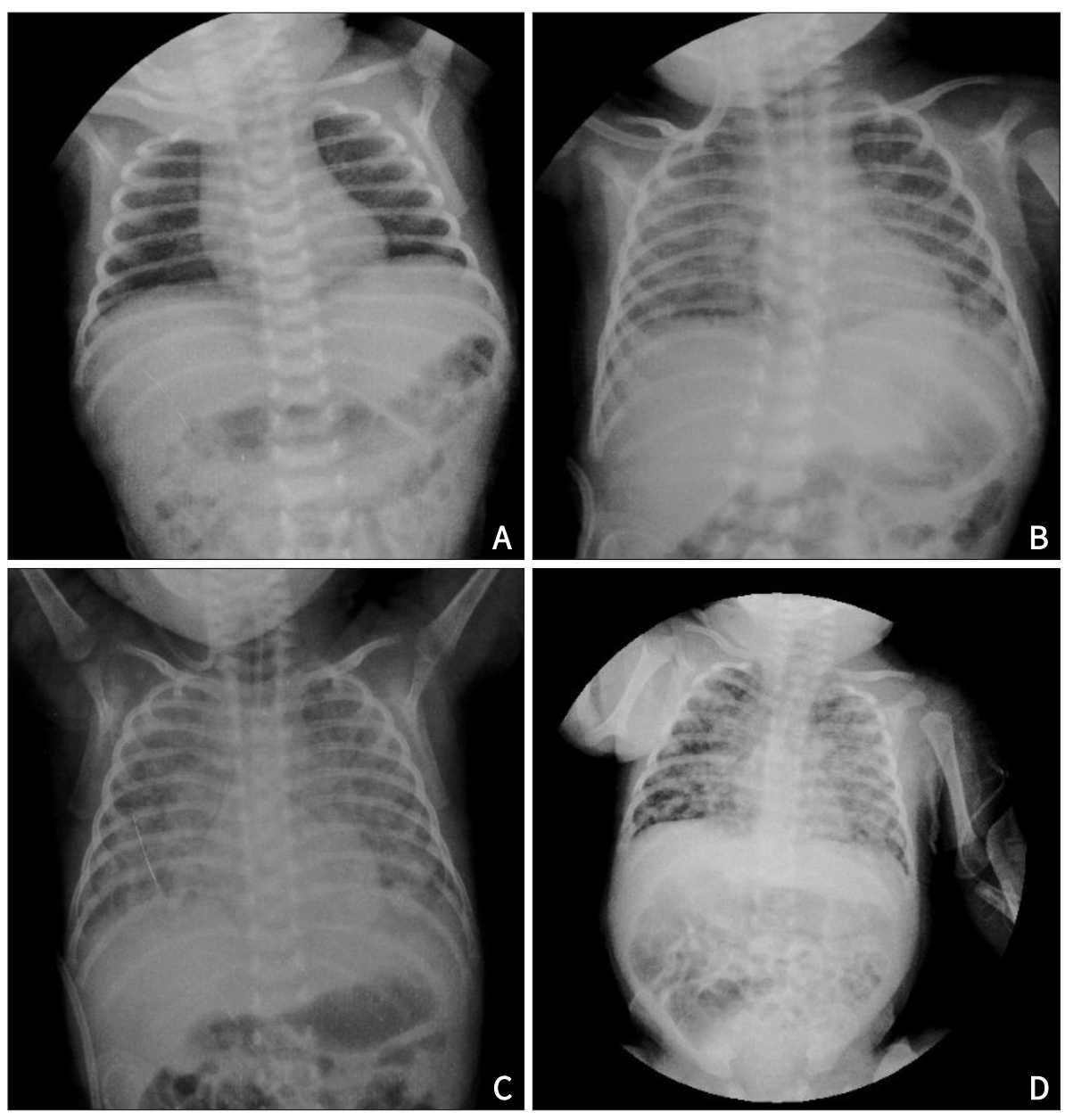

Fig. 1. Normal chest radiograph on day of life 15 (A). Bilateral hazy pulmonary opacity on chest radiograph on day of life 21 (B). Increased hazy density in both lung fields on a chest radiograph on day of life 35 (C). Bilateral nodular opacity on chest radiograph on day of life 43 (D). 
of $10,600 / \mathrm{mm}^{3}$, platelet count of $262,000 / \mathrm{mm}^{3}$, and CRP of $1.4 \mathrm{mg} / \mathrm{dL}$. A slight increase in the CRP level was also observed. The cerebrospinal fluid examination performed on the same day showed normal results. (white blood cell $1 / \mathrm{mm}^{3}$, protein $62.6 \mathrm{mg} / \mathrm{dL}$, glucose $47 \mathrm{mg} / \mathrm{dL}$ ) Cultures of urine, blood, and cerebrospinal fluid were all normal. No specific causative bacterial organisms or viruses were detected in the polymerase chain reaction of respiratory tract causative organisms. Echocardiography and abdominal ultrasonography performed on the same day were all normal. She had no symptoms of hepatosplenomegaly and abdominal distension.

On the $24^{\text {th }}$ day of life, chest radiographs showed increased haziness in both lungs (Fig. 1B). CRP increased to $4.4 \mathrm{mg} / \mathrm{dL}$. The medical staff assumed sepsis and bacterial pneumonia, and cefotaxime and vancomycin were administered from the 24th day after birth. Intravenous immunoglobulin was administered at $500 \mathrm{mg} / \mathrm{kg} /$ dose once daily for 4 days. On $25^{\text {th }}$ day of life, CRP peaked at $8.0 \mathrm{mg} / \mathrm{dL}$ and decreased to $1.6 \mathrm{mg} / \mathrm{mL}$ on the $31 \mathrm{st}$ day of life. The respiratory rate normalized to $45 / \mathrm{min}$, but the apnea persisted. On the 35th day after birth, despite nasal continuous positive airway pressure treatment, the oxygen saturation decreased to $83 \%$, and the patient was slightly lethargic, tachypnea re-occurred with a respiratory rate of more than $80 / \mathrm{min}$. In the chest radiographs examined that day, infiltration aggravation was observed in both lungs (Fig. 1C). The CRP level was also elevated to 9.1 on the 36th day of life. Medical staff suspected pneumonia caused by resistant bacteria or pneumonia caused by a fungal infection. The medical staff changed the antibiotics to vancomycin, clindamycin, and amikacin, and administered fluconazole to the suspected fungal infection. It was difficult to completely rule out the possibility of bronchopulmonary dysplasia due to the worsening of apnea and dyspnea despite antibiotic and antifungal treatment. thus dexamethasone was administered at 0.35 mg twice daily for 2 weeks. A second intravenous immunoglobulin was also administered at $500 \mathrm{mg} / \mathrm{kg} /$ dose once daily for 4 days. After steroid treatment, respiratory distress showed improvement, mechanical ventilation was stopped and the amount of feeding was increased.

On the 50th day after birth, but fever occurred above $38^{\circ} \mathrm{C}$. Even when oxygen was supplied with a high-flow nasal cannula, the number of breaths was more than 90 times, and chest radiographs showed that infiltration was increased in both lungs (Fig. 1D). In addition, CRP was still high at 7.9 $\mathrm{mg} / \mathrm{dL}$. The antibiotics were replaced with vancomycin and imipenem, and a third immunoglobulin was administered for 4 days. The fever was checked at approximately $38^{\circ} \mathrm{C}$ once a day until the 65 th day after birth.

On the 58th day of life, a chest CT scan showed multifocal nodular and patchy consolidation in both lung parenchyma (Fig. 2A and 2B). On the 63rd day after birth, the mother of the infant visited the emergency room complaining of fever and decreased consciousness, and was diagnosed with tuberculous meningitis by cerebrospinal fluid examination. Chest computotomography showed suspicion of tuberculosis with bronchiolitis and Quantiferon-TB known as interferon-gamma release assay (IGRA) and sputum acid-fast bacillus (AFB) test was positive. The twin mothers started treatment with anti-tuberculosis drugs and were isolated.

On the same day, the second twin, who was in good condition during hospitalization, had a fever and was admitted to the hospital. At the time of admission, he did not have any shortness of breath other than fever. He had previously been discharged on the 36th day after birth. And one

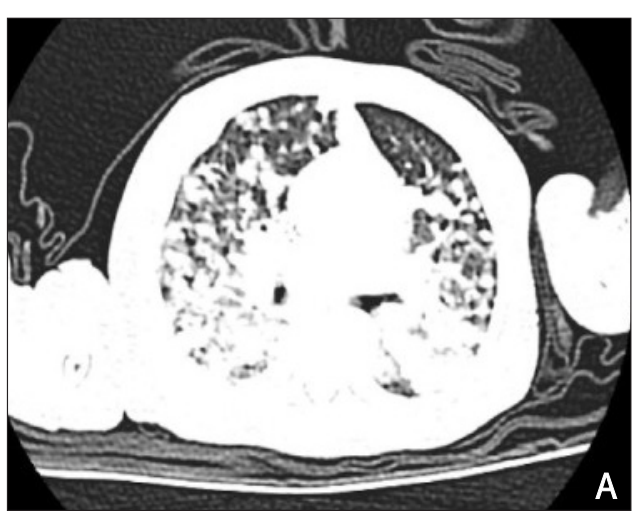

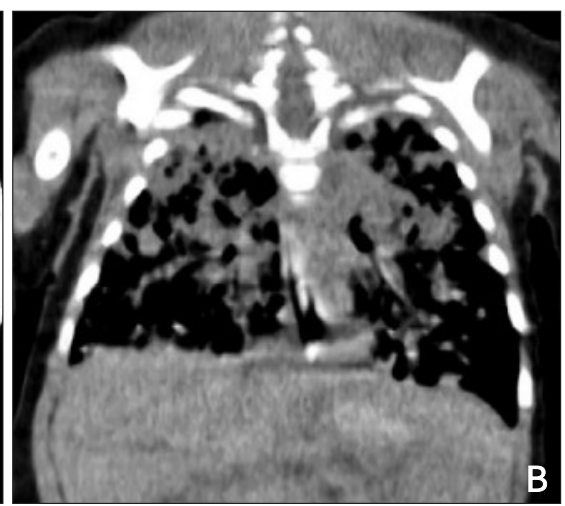
Big. 2. Multifocal nodular and patchy consoli-
dation on CT scans. 
week after discharge, he was in good condition, so Bacillus Calmette-Guerin vaccination was administered intradermally. He had no local manifestations at vaccination site and no lymphadenopathy. The first twin was hospitalized, so Bacillus Calmette-Guerin vaccination was not administered. A chest radiograph showed consolidation in the right upper and middle lobes, as well as numerous tiny nodular densities in both lungs. Medical staff performed AFB staining and sputum TB PCR tests on the gastric fluid samples of twins. Mycobacterium tuberculosis was detected in the acid-fast bacillus (AFB) stain, TB polymerase chain reaction (PCR), and TB culture of the twins. The twins were diagnosed with congenital tuberculosis, and anti-tuberculosis treatment was initiated. Anti-tuberculosis drugs include isoniazid, rifampin, pyrazinamide, and ethambutol. After administration of anti-tuberculosis drugs, clinical symptoms such as fever and dyspnea improved. The patients were discharged together from the hospital on the 79th day of life and were followed up on an outpatient basis. During follow-up after discharge from the hospital, the 122-day-old first child was admitted to the outpatient clinic because of dyspnea and grunts. The second child was fine though. Threrfore, echocardiography was performed on an outpatient basis. Echocardiography showed a tricuspid regurgitation pressure difference of 55 $\mathrm{mmHg}$ with dilatation of the right atrium and right ventricle on echocardiogram (Fig. 3). The medical staff determined that the patient's shortness of breath was due to pulmonary arterial hypertension. Immediately after admission to the intensive care unit, the heart rate decreased with apnea. Be-

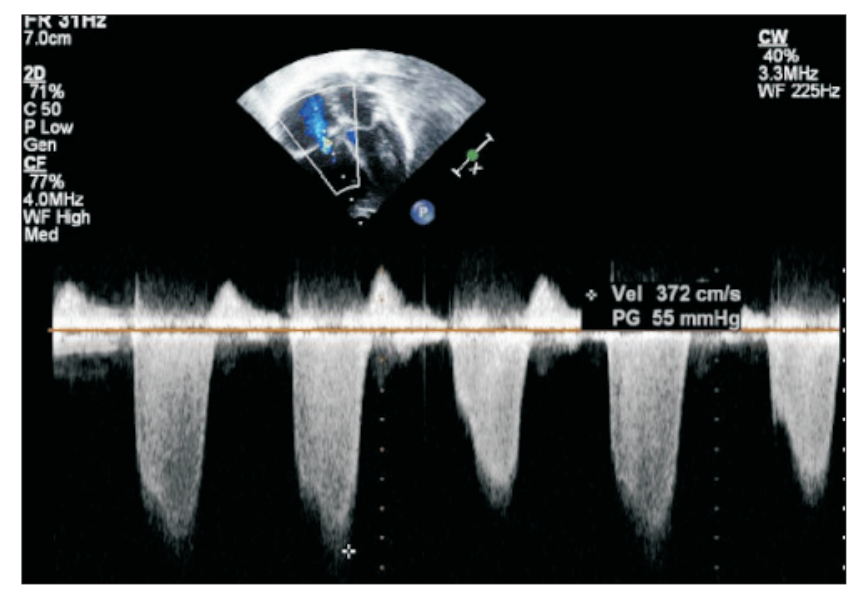

Fig. 3. Echocardiography showed a tricuspid regurgitation pressure difference of $55 \mathrm{mmHg}$ with dilatation of the right atrium and right ventricle. fore starting mechanical ventilation. The patient's vital signs worsened rapidly before blood tests. There was no spontaneous breathing, heart rate was 40 beats per minute, and oxygen saturation was checked at 40. CPR was performed, but the patient died on the day of admission.

\section{DISCUSSION}

Congenital tuberculosis is a rare disease with a mortality rate of up to $44 \%$. Symptoms are non-specific and appear at 2-3 weeks of age [5]. The most common clinical signs were hepatomegaly (75\%), dyspnea (72\%), fever (48\%), lymphadenopathy (38\%), abdominal distension (24\%), lethargy or irritability (21\%), ear discharge (17\%) and papular eruption (14\%) [6]. The non-specific symptoms of congenital tuberculosis often mimic more common neonatal disease such as bacterial sepsis or congenital viral infection [5]. In a systematic review of 92 cases, 55 patients (59.78\%) were misdiagnosed. The misdiagnoses included neonatal pneumonia in 49 patients (89.09\%), neonatal sepsis in 18 patients (32.73\%), neonatal hepatitis syndrome in three patients (5.45\%) and anemia in one patient (1.82\%) [6]. Our patient was initially misdiagnosed with bacterial pneumonia.

Congenital tuberculosis infection occurs after the maternal infection precedes. The infection route of congenital tuberculosis is directly spread through the umbilical cord, the swallowing or aspiration of infected amniotic fluid, and direct contact with an infected genital lesion of the mother during delivery [7].

Several studies also have investigated the prognostic factors of congenital tuberculosis. In a retrospective study of 170 children with congenital tuberculosis, mortality was not influenced by prematurity, liver dysfunction, thrombocytopenia, or disseminated intravascular coagulation. Infants with intracranial lesions had a higher mortality rate than those without intracranial lesions. Infants who presented with symptoms after 3 weeks of age had a higher survival rate. Miliary patterns and multiple pulmonary nodule patterns on chest imaging are associated with a poor prognosis like the first twin of our case. This may be due to the miliary pattern, and the pattern of multiple pulmonary nodules on chest film reflects a disseminated disease [1].

In addition, the causes of high mortality are lack of awareness, delay in diagnosis and treatment, and rap- 
id progression of the disease. Therefore, early diagnosis and treatment are important. In a study by Peng et al., the overall mortality rate was $43.48 \%$, but the mortality rate of congenital tuberculosis was only $15.38 \%$ after diagnosis and anti-tuberculosis treatment. In a study by Li et al., 68 of 169 patients (40\%) died. All 40 patients without treatment died from congenital tuberculosis, however, of the 129 children treated with anti-tuberculosis treatment, only 28 (19\%) died [6.

There are various causes of deaths. In the analysis of 92 case reports, 40 patients (43.48\%) died. Among the 40 children who died, the cause of death included respiratory failure in $26(65 \%)$, multiple organ dysfunction syndrome in $3(7.5 \%)$, intracranial hemorrhage in $1(2.5 \%)$ and unknown causes in 10 (25\%). In the patient case, the patient died of pulmonary arterial hypertension caused by congenital tuberculosis.

To avoid delay in diagnosis, it is necessary to consider the diagnosis of congenital tuberculosis in the following situations. First, congenital tuberculosis should be suspected in infants under 2 months of age who have pneumonia or sepsis that do not respond to antibiotic treatment. In particular, suspicion should be raised if pneumonia and hepatosplenomegaly are present [6]. Second, infants have respiratory distress, hepatosplenomegaly and fever during the first three months after birth [1]. Third, multiple pulmonary nodules and miliary patterns were observed on chest imaging 4 weeks post-natal. Fourth, multiple focal lesions in the liver and spleen are shown in abdominal imaging findings. Fifth, mothers had active tuberculosis during pregnancy [1]. In a study analyzing 170 infants with congenital tuberculosis, 121 mothers were diagnosed with tuberculosis after childbirth. Of these, 39 mothers were asymptomatic and were not diagnosed with tuberculosis until their babies were diagnosed with congenital tuberculosis [1]. In another study, seven mothers $(9.86 \%)$ were diagnosed with tubercu- losis before pregnancy, 16 (22.54\%) were diagnosed during pregnancy and 48 (67.6\%) were diagnosed after giving birth [6]. Most reported cases were diagnosed after delivery rather than when the mother was diagnosed with tuberculosis before delivery. As in our case, it is common for a child to be diagnosed after symptom onset. Even if the mother is not diagnosed with active tuberculosis, it is important to consider tuberculosis in the clinical situations listed above.

The possibility of congenital tuberculosis, although rare, should be considered when a newborn has pneumonia that does not respond to antibiotic treatment.

\section{CONFLICT OF INTEREST}

No potential conflict of interest relevant to this article was reported.

\section{REFERENCES}

1. Peng W, Yang J, Liu E. Analysis of 170 cases of congenital TB reported in the literature between 1946 and 2009. Pediatr Pulmonol 2011;46:1215-24.

2. Kim JS, Shin JY, In HK, Shim EH. Review on global burden of tuberculosis in 2019. Public Health Wkly Rep, PHWR 2020; 13:3161-75.

3. Parslow R, El-Shimy NA, Cundall DB, McKinney PA. Tuberculosis, deprivation, and ethnicity in Leeds, UK, 1982-1997. Arch Dis Child 2001;84:109-13.

4. World Health Organization. Global tuberculosis report 2020. Geneva: World Health Organization; 2020. p. 227.

5. Mazade MA, Evans EM, Starke JR, Correa AG. Congenital tuberculosis presenting as sepsis syndrome: case report and review of the literature. Pediatr Infect Dis J 2001;20:439-42.

6. Li C, Liu L, Tao Y. Diagnosis and treatment of congenital tuberculosis: a systematic review of 92 cases. Orphanet J Rare Dis 2019;14:131.

7. Gleason CA, Juul SE. Avery's diseases of the newborn. 10th ed. Philadelphia: Elsevier; 2018. p. 527-52. 